Article

\title{
Encapsulation of Dual Emitting Giant Quantum Dots in Silica Nanoparticles for Optical Ratiometric Temperature Nanosensors
}

\author{
Elisabetta Fanizza ${ }^{1,2, * \mathbb{D}}$, Haiguang Zhao ${ }^{3}$, Simona De Zio ${ }^{1,+}{ }^{,}$Nicoletta Depalo ${ }^{2, *(D)}$, \\ Federico Rosei ${ }^{4}$, Alberto Vomiero ${ }^{5,6}$ (D) M. Lucia Curri ${ }^{1,2}$ and Marinella Striccoli ${ }^{2}$ (D) \\ 1 Dipartimento di Chimica, Università degli Studi di Bari, Via Orabona 4, 70126 Bari, Italy; \\ simona.dezio2@unibo.it (S.D.Z.); marialucia.curri@uniba.it (M.L.C.) \\ 2 CNR-IPCF, SSO Bari, Via Orabona 4, 70126 Bari, Italy; m.striccoli@ba.ipcf.cnr.it \\ 3 State Key Laboratory of Bio-Fibers and Eco-Textiles, College of Physics, Qingdao University, \\ No. 308 Ningxia Road, Qingdao 266071, China; hgzhao@qdu.edu.cn \\ 4 Centre Énergie, Matériaux et Télécommunications, Institut National de la Recherche Scientifique, \\ 1650 Boul. Lionel Boulet, Varennes, QC J3X 1S2, Canada; rosei@emt.inrs.ca \\ 5 Division of Materials Science, Department of Engineering Sciences and Mathematics, \\ Luleå University of Technology, SE-971 98 Luleå, Sweden; alberto.vomiero@ltu.se \\ 6 Department of Molecular Sciences and Nanosystems, Ca' Foscari University of Venice, Via Torino 155, \\ 30172 Venezia Mestre, Italy \\ * Correspondence: elisabetta.fanizza@uniba.it (E.F.); n.depalo@ba.ipcf.cnr.it (N.D.) \\ † Dipartimento di Chimica “Giacomo Ciamician”, Università degli Studi di Bologna, Via Selmi 2, \\ Bologna, Italy.
}

Received: 30 March 2020; Accepted: 14 April 2020; Published: 16 April 2020

\begin{abstract}
Accurate temperature measurements with a high spatial resolution for application in the biomedical fields demand novel nanosized thermometers with new advanced properties. Here, a water dispersible ratiometric temperature sensor is fabricated by encapsulating in silica nanoparticles, organic capped PbS@CdS@CdS "giant" quantum dots (GQDs), characterized by dual emission in the visible and near infrared spectral range, already assessed as efficient fluorescent nanothermometers. The chemical stability, easy surface functionalization, limited toxicity and transparency of the silica coating represent advantageous features for the realization of a nanoscale heterostructure suitable for temperature sensing. However, the strong dependence of the optical properties on the morphology of the final core-shell nanoparticle requires an accurate control of the encapsulation process. We carried out a systematic investigation of the synthetic conditions to achieve, by the microemulsion method, uniform and single core silica coated GQD $\left(\mathrm{GQD} @ \mathrm{SiO}_{2}\right)$ nanoparticles and subsequently recorded temperature-dependent fluorescent spectra in the 281-313 K temperature range, suited for biological systems. The ratiometric response- the ratio between the two integrated PbS and CdS emission bands-is found to monotonically decrease with the temperature, showing a sensitivity comparable to bare GQDs, and thus confirming the effectiveness of the functionalization strategy and the potential of GQD@SiO ${ }_{2}$ in future biomedical applications.
\end{abstract}

Keywords: QD functionalization; silica shell; optical sensor; ratiometric sensing; nanothermometers

\section{Introduction}

Temperature represents an essential physical property in multiple fields. In particular, in the biomedical field, it is a reliable indicator of the occurrence of active intracellular chemical reactions, also able to provide basic information useful for understanding specific physiological conditions 
and for assisting early diagnosis and therapy. Temperature influences the equilibrium constants and biochemical reaction kinetics in fundamental cell processes, including enzyme activity, gene expression, cell division and energy metabolism [1-4]. In cases of diseases such as cancer, pathological studies have revealed that malignant cells in tissues change their metabolic activities, leading to acute deviation of the intracellular temperatures from the normal state [1-4]. Furthermore, accurate information on the local temperature profile is also relevant in carrying out therapeutic treatments, such as phototherapy, for the selective and local killing of diseased cells, to avoid unwanted destruction of the healthy tissue surrounding the treated area. Since temperature variation inside cells occurs typically at a very small length scale [5], conventional thermal sensors based, for example, on thermocouples cannot be effectively applied due to their low spatial resolution. They generally operate in the contact mode, thus requiring a challenging fabrication procedure to miniaturize thermometers down to nanometer regime for temperature detection in cells. Such essential requirements have stimulated research on the design and development of novel nanoscale temperature sensors [6], able to provide an accurate and sensitive temperature measurement with high spatial resolution. Different types of nanothermometers [7-12] have been developed that offer a direct read-out of the temperature by transducing a temperature-dependent change of an optical property of the selected material, such as absorption, emission or Raman scattering. In this regards, fluorescent nanoparticles (NPs), such as quantum dots (QDs) [9,13,14], luminescent semiconductor [15], carbon dots [12,16], rare earth doped up-converting or down-converting NPs $[1,7,8,17,18]$, polymeric particles $[2,3,11,19]$ or organic dyes [10] are emerging as promising luminescence nanothermometry devices. They take advantage of the thermally induced changes of a fluorescence characteristic such as band intensity, band shape, spectral position and lifetime for a precise, and often multiparametric and ratiometric, $[3,7,9,10,20]$ temperature detection. Due to the complex cellular environment, fluorescent temperature sensors for in vitro measurements need to be bright enough to overcome the background signals and the autofluorescence from cellular materials, being, at the same time, non-toxic for the cells in long term cellular studies.

Inorganic semiconductor QDs, characterized by a high quantum yield and superior photostability, represent fluorescent systems more robust than organic dyes that, conversely, suffer from too fast fluorescence bleaching, thus appearing inappropriate for long term monitoring [21]. Recent advances in solution phase synthesis of luminescent QDs have provided access to nanoheterostructures with multiple states $[9,22-25]$ capable of efficient light emission of different colors, and with great potential for their use in ratiometric sensors. Ratiometric fluorescent probes are, in general, more robust and reliable than nonratiometric ones, which monitor a change of a single fluorescence signal. In the field of temperature sensors, the presence of two (or more) emission bands and the ability to establish a defined relationship between their emission properties, over the investigated temperature range, advantageously result in a more sensitive, concentration-independent, temperature detection. Furthermore, dual emission-systems do not suffer from environmental interference, overcoming limitations arising from inaccurate detection due to the effect on the photoluminescence of refractive index, surrounding environment, presence of quenching agent [9]. Zhao et al. [9,26-28] have reported the fabrication of "giant" PbS@CdS@CdS QDs (GQDs) where the PbS core produces an emission band in the near infrared (NIR) region and the CdS shell in the visible portion of the electromagnetic spectrum. Due to the "giant" CdS shell (from $1.5 \mathrm{~nm}$ up to several tens $\mathrm{nm}$ ), these optically active GQDs exhibit very high photostability towards photobleaching, even under continuous laser excitation, and have been demonstrated to be efficient temperature ratiometric and multiparametric probes [9]. However, being only dispersible in organic solvents, due to the hydrophobic nature of their surface, an appropriate surface functionalization must be developed as a valuable route to improve the GQDs dispersibility in aqueous environment and, at the same time, to make them biocompatible, hence suited for further application as thermal sensors in biological media. This approach may also potentially reduce the release of the heavy metals components forming the GQDs outside, decreasing the nanostructure's toxicity. 
Among the different functionalization strategies developed to ensure that QDs and NPs are water dispersible and covered by biocompatible matrix, we recall encapsulation in molecular micelles or lipid-based structures [29,30], polymeric [31] or inorganic shell coating [32-35]. Here we chose the growth of a hydrophilic silica shell, as it represents a versatile and low cost approach [32-34]. The silica shell has been reported to be chemically stable in water, although solubility depends on the silica structure and solution $\mathrm{pH}$, it has a low cytotoxicity and can be easily chemically modified [35-37]. A silica coating can limit diffusion of toxic elements outside and, meanwhile, reduce oxygen permeation inwards, ultimately protecting the core from oxidation reaction, and thus preventing the degradation of its optical properties [38]. In addition, the silica shell, being optically transparent in the visible range, brings a limited contribution to the absorption, which is a critical requirement for being integrated in a fluorescence based temperature sensor [39]. However, a silica shell growth process presents several issues concerning the structure and the properties of the final NP [32-34]. In general, while single core nanostructures preserve the optical properties of the core, the formation of multiple cores entrapped within a single silica particle, in close proximity with each other, may result in detrimental absorption broadening, fluorescent shift and quenching. Besides core multiplicity, also the formation of an inhomogeneous silica coating and the occurrence of aggregated structures needs to be prevented to preserve the optical properties of the emitting core and convey them to the obtained water dispersible heterostructures, able to limit any release of metal ion in the surrounding environment.

For this purpose, we exploit here a microemulsion system to encapsulate organic capped PbS@CdS@CdS GQDs, already shown to act as effective ratiometric temperature sensors, into silica NPs and we systematically investigate the experimental conditions for achieving a uniform silica shell. Silica growth parameters have been controlled, adjusting both surface chemistry of the GQD core and the GQD/silica precursor molar ratio. Subsequently, surface reactive amino groups have been grafted onto the silica surface to provide suitable anchoring points for further possible bioconjugation reactions. The resulting core-shell nanostructures still feature the dual emission in the visible and NIR region, ascribed to the giant $\mathrm{CdS}$ shell and $\mathrm{PbS}$ core domains, respectively, characteristic of the inorganic GQDs. Emission spectra, recorded over a temperature range relevant for biological systems, confirm the monotonic decrease of the ratiometric fluorescence with the temperature with the same sensitivity measured for bare GQDs [9]. The overall results clearly show that encapsulation of GQDs in silica NPs, while making them dispersible in aqueous media, safely preserve their properties and, hence, function as fluorescence thermal sensors to be applied in health applications.

\section{Materials and Methods}

Materials. For the synthesis of the giant PbS@CdS@CdS QDs the following chemicals were used: lead (II) chloride $\left(\mathrm{PbCl}_{2}, 98 \%\right.$ ), oleylamine (OLA, technical grade, 70\%), oleic acid (OA, 90\%), cadmium oxide $(99 \%$, powder, $\mathrm{CdO})$, cadmium acetate $\left(\mathrm{Cd}(\mathrm{OA})_{2}, 99,9995 \%\right)$ and sulfur $(99.9999 \%$ powder, $\mathrm{S})$. The silica shell growth and functionalization required tetraethyl orthosilicate $(98 \% \mathrm{~d}=0.934 \mathrm{~g} / \mathrm{mL}$, TEOS), aqueous ammonia $\left(\mathrm{d}=0.900 \mathrm{~g} / \mathrm{mL}, \mathrm{NH}_{4} \mathrm{OH}\right), 5$-polyoxyethylene nonylphenylether (Igepal CO-520, Mn = 441) and (3-Aminopropyl)triethoxysilane (97\%, APS). For the ninhydrin test, ninhydrin and 2,6-lutidine (98\%, Alfa Aesar) were used. All chemicals, purchased from Sigma Aldrich @ Merck KGaA, Darmstadt, Germany, if not specified otherwise, were used without purification. All solvents, namely methanol, ethanol, chloroform, cyclohexane, octadecene (ODE) were of the highest purity available.

Synthesis of GQDs. Synthesis of PbS@CdS QDs was carried out via a two-step cation exchange reaction, as reported in $[26,40]$, followed by the growth of a thick CdS shell. In particular, the PbS cores were synthesized by using OLA as ligands [40]. Typically, $\mathrm{PbCl}_{2}(3.6 \mathrm{mmol})$ in OLA $(2.4 \mathrm{~mL})$ and sulfur $(0.36 \mathrm{mmol})$ in OLA $(0.24 \mathrm{~mL})$ were purged, respectively, by $\mathrm{N}_{2}$ at room temperature for $30 \mathrm{~min}$. The $\mathrm{PbCl}_{2}$-OLA suspension was heated and kept at $160{ }^{\circ} \mathrm{C}$ for $1 \mathrm{~h}$ and then cooled down to $120^{\circ} \mathrm{C}$ under vacuum for $15 \mathrm{~min}$ and then put under $\mathrm{N}_{2}$ flux. The sulfur in OLA at room temperature was quickly injected into the $\mathrm{PbCl}_{2}$-OLA suspension, the solution was vigorously stirred and the reaction 
was conducted at $100^{\circ} \mathrm{C}$ for $10 \mathrm{~min}$, before being quenched by putting the reaction flask into cold water. Ethanol/acetone (1:1) was added, and then the suspension was centrifuged and the QDs pellets were dispersed in toluene. PbS@CdS QDs with a thin shell were synthesized via a cation exchange method [40]. Typically, CdO (2.3 mmol), OA (2 mL) and ODE (10 mL) were heated to $255^{\circ} \mathrm{C}$ under $\mathrm{N}_{2}$ for $30 \mathrm{~min}$. The clear solution was cooled down to $155^{\circ} \mathrm{C}$ under vacuum for $15 \mathrm{~min}$ and then put under $\mathrm{N}_{2}$. PbS QDs suspension in toluene $(1 \mathrm{~mL}$, absorbance $=3$ at the first exciton peak $)$ was diluted in $10 \mathrm{~mL}$ toluene, degassed for $30 \mathrm{~min}$ and then immediately heated to $100^{\circ} \mathrm{C}$. The $\mathrm{Cd} / \mathrm{OA}$ mixture was injected and the reaction was let to proceed at $100^{\circ} \mathrm{C}$ for $120 \mathrm{~min}$ and at $240^{\circ} \mathrm{C}$ for $2 \mathrm{~h}$. The reaction was quenched by injection of cold toluene $\left(-20^{\circ} \mathrm{C}\right)$, and ethanol was added to collect the PbS@CdS QDs finally dispersed in hexane. Deposition of the thick CdS layer on PbS@CdS QDs followed the successive ionic layer absorption and reaction (SILAR) procedure described by Zhao et al. $[9,26]$. Typically, in a $100 \mathrm{~mL}$ round-bottom flask, OLA (5 mL), ODE (3 mL) and PbS@CdS QDs $\left(2 \times 10^{-7} \mathrm{~mol}\right.$ in hexane) were degassed at $110^{\circ} \mathrm{C}$ for $20 \mathrm{~min}$. The reaction flask was re-stored with $\mathrm{N}_{2}$ and the temperature was further raised to $240{ }^{\circ} \mathrm{C}$ with stirring. The sulfur dispersed in ODE $(1 \mathrm{~mL}, 0.2 \mathrm{M})$ was added dropwise and the mixture allowed to react for $10 \mathrm{~min}$, followed by dropwise addition of $1 \mathrm{~mL} 0.2 \mathrm{M} \mathrm{Cd}(\mathrm{OA})_{2}$ in ODE. The shell was then annealed for $90 \mathrm{~min}$. All subsequent shells were annealed at $240{ }^{\circ} \mathrm{C}$ for $1.5 \mathrm{~h}$ following the injection of sulfur and $2 \mathrm{~h}$ following dropwise addition of the $\mathrm{Cd}(\mathrm{OA})_{2}$ in $\mathrm{ODE}$. Sulfur/Cd(OA $)_{2}$ addition volumes for shell addition cycles 1-8 were as follows: 1, 1, 1.5, 1.5, 2, 2, 3 and $3 \mathrm{~mL}$, respectively. The reaction was quenched by injection of cold toluene $\left(-20^{\circ} \mathrm{C}\right)$. Ethanol was added, then the suspension was centrifuged and the supernatant was removed. The QDs were dispersed in hexane.

Growth of the silica shell on the GQDs. Silica shell was grown onto the GQDs by using a base assisted water-in-oil microemulsion approach. [41,42] A preliminary treatment of GQDs with OLA was carried out by stirring the GQDs solution with an excess of neat OLA at a room temperature for $12 \mathrm{~h}$ and collect them by ethanol non solvent addition and redispersion in cyclohexane. Sequentially, $350 \mu \mathrm{L}$ of IGEPAL CO-520, $200 \mu \mathrm{L}$ of $\mathrm{NH}_{4} \mathrm{OH}$ and a varied volume of TEOS (in the range of 20-100 $\mu \mathrm{L}$ ) were injected to $6 \mathrm{~mL}$ of a cyclohexane solution of GQDs, at different concentrations. The microemulsion was then kept under vigorous stirring at controlled temperature $\left(28^{\circ} \mathrm{C}\right)$ for $18 \mathrm{~h}$. Functionalization with APS was carried out by adding $600 \mu \mathrm{L}$ of APS to the microemulsion, stirred overnight at $28^{\circ} \mathrm{C}$. Amino-functionalized GQD@SiO ${ }_{2}$ NPs were collected by adding methanol, which is able to disrupt the micelle, and centrifuged at $7800 \times g$ for 20 min (Beckman J2-21). Repeated cycles of NP dispersion in ethanol and centrifugation were carried out to completely remove surfactant residuals and to finally collect NPs, finally dispersed in $2 \mathrm{~mL}$ of ethanol. The effective characterization and quantitative analysis of primary amino groups in a different type of systems [43], was carried out by a ninhydrin assay. A ninhydrin solution was prepared by dissolving $110 \mathrm{mg}$ of ninhydrin in $16 \mathrm{~mL}$ of ethanol $(0.68 \% \mathrm{w} / \mathrm{v})$ and adding $4 \mathrm{~mL}$ of 2,6 lutidine. A preliminary calibration experiment was performed by adding an excess of ninhydrin solution in ethanol to APS standard solutions ranging from $50 \mu \mathrm{M}$ to $15 \mathrm{mM}\left(\varepsilon=2678 \pm 117 \mathrm{~L} \mathrm{~mol}^{-1} \mathrm{~cm}^{-1}\right)$. Of ninhydrin solution $(0.03 \mathrm{M}) 1.9 \mathrm{~mL}$ was added to $0.1 \mathrm{~mL}$ of GQD@SiO $\mathrm{NPs}_{2}$ collected after the functionalization reaction. The suspension was then heated up to $90^{\circ} \mathrm{C}$ for $5 \mathrm{~min}$. The fact that the solution's color changes from the yellow (color of the free ninhydrin) to blue (color of the Ruhemann's blue reaction by-product) accounts for the presence of amino groups and the absorbance intensity measured at $570 \mathrm{~nm}$, characteristic of the Ruhemann's by-product, was used to titrate the amount of amino groups.

Characterization techniques. Transmission electron microscopy (TEM) analysis was performed by using a JEOL 100 (JEOL Italia S.P.A, Milan, Italy), operating at $100 \mathrm{kV}$ and equipped with a W electron source and a CCD high resolution camera. Deposition of the NPs was carried out by dipping a carbon coated copper grid in the sample suspension and let the solvent evaporate. Size statistical analysis was carried out by measuring average NPs size and determining relative standard deviation $(\sigma \%)$ for more than 100 NPs of each sample. UV-Vis absorption spectra were recorded with a Cary 5000 (Varian) UV/Vis/NIR spectrophotometer (Agilent Technologies Italia, Milan, Italy). Fluorescent 
spectra were taken with a Fluorolog-3 system (Horiba Jobin Yvon Burlington, Canada) equipped with a THMS 600 temperature-controlled stage to monitor variation of photoluminescence (PL) spectra in the temperature range $282-313 \mathrm{~K}$.

\section{Results and Discussion}

Here, the base assisted water-in-oil microemulsion method was exploited to coat organic dispersible OLA-OA capped GQDs with a silica shell, in order to make them dispersible in aqueous media, thus enabling their possible application in the biomedical field. The interest towards such a GQDs system arises from their original fluorescent properties, able to provide an accurate read-out of the temperature with ultrahigh sensitivity [9] in a wide temperature range, thus being promising as fluorescent nanothermometry systems.

Prepared by a solution phase approach, GQDs consist of a PbS core, synthesized by using a hot injection procedure and coated with a thin CdS shell obtained by cation exchange, and finally with a thick CdS shell achieved by SILAR in the presence of OLA and $\mathrm{Cd}(\mathrm{OA})_{2}$ (Figure 1A). As reported by Zhao et al. [26], this procedure results in PbS@CdS@CdS structures with a PbS core size estimated to be $1.2 \mathrm{~nm}$, a thin interlayer of zinc blend CdS and a thick outer shell of wurtzite CdS. As shown in Figure 1B, quite monodispersed triangular and asymmetric shaped NPs with an average final size of $15 \mathrm{~nm}(\sigma \%=11 \%$, Figure 1A) have been achieved [26].
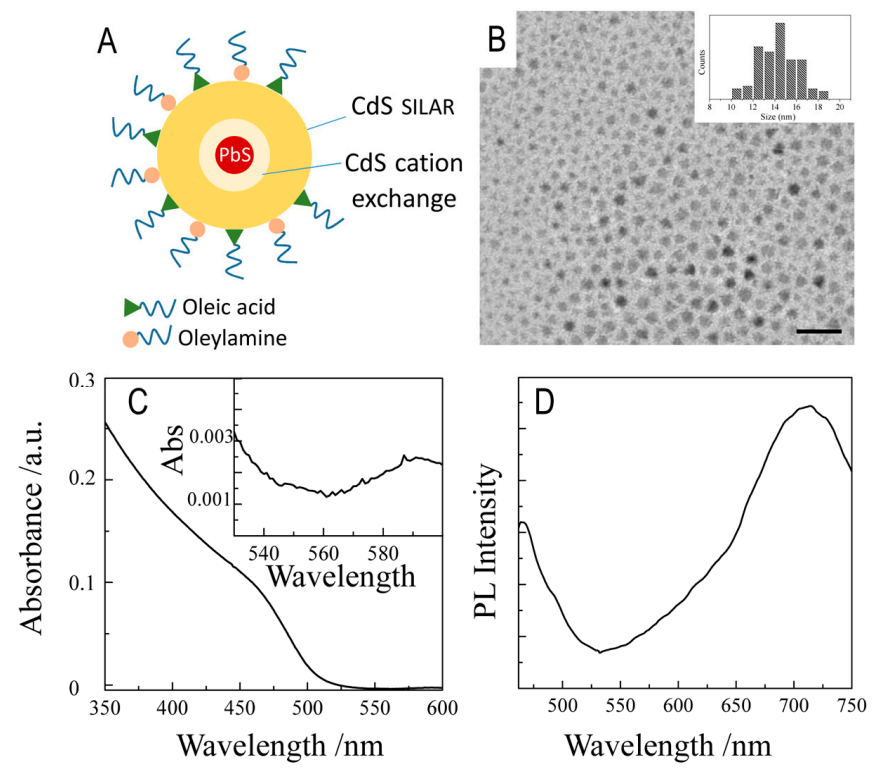

Figure 1. Sketch of the giant PbS@CdS@CdS NCs (A) and TEM micrograph (B, scale bar 50 nm) with size statistical analysis (B, inset), UV-Vis absorption (C) and photoluminescence (D, $\lambda$ ex $=400 \mathrm{~nm}$, $\mathrm{T}=294 \mathrm{~K}$ ) spectra of the core-shell-shell sample. The absorbance profile in the 540-600 nm wavelength range has been enlarged in the inset of panel (C).

The UV-Vis absorption profile is mainly dominated by the excitonic transition of the thick CdS shell, which appeared as a shoulder at nearly $470 \mathrm{~nm}$, with an onset at $512 \mathrm{~nm}$ (Figure 1C), and a very weak absorption feature at $591 \mathrm{~nm}$ (Figure 1C, inset), which could be related to the very small PbS core [26]. A clearer contribution of the PbS domain can be observed in the photoluminescence spectrum of the GQDs (Figure 1D), which shows the typical dual emission characteristic of the nano-heterostructures [9,26-28]. The broad and intense band centered at $715 \mathrm{~nm}$ in the near infrared (NIR) region can be assigned to the PbS core, usually characterized by a large Stokes shift (here nearly $364 \mathrm{meV}$ ), while the band at $465 \mathrm{~nm}$, in the visible region of the spectrum, was generated by the CdS thick shell, being the peak position in agreement with the band gap energy of the bulk CdS. The lower 
emission intensity of the CdS domain can be reasonably ascribed to trap band states and thus to the non-radiative recombination processes, increasing with the number of the SILAR deposition cycles [26].

Being these GQDs only dispersible in apolar organic solvents, to allow their application in a biological environment, they need to be made dispersible in aqueous media by means of a suitable surface functionalization.

Direct ligand exchange [44], encapsulation in self assembled molecular structures [29,30,45], polymer coating [31] and silica shell coating [32-34] have been reported as a possible strategy for the phase transfer of organic-capped NPs in water. Here, silica shell growth is used. The low toxicity and chemical stability of the silica structure, [46] which undergoes to dissolution only at acidic $\mathrm{pH}$ [47], and the easy integration of surface functional groups, needed for bioconjugation and site specific recognition reactions, make silica shell a suited biocompatible coating, able to limit any leakage in the surrounding environment of the heavy metals forming the GQDs.

The water-in-oil microemulsion approach, extensively reported as a strategy used to coat with a hydrophilic siloxane network organic capped NPs [32-34,41,42,48], was exploited to grow the silica shell onto the GQDs. As described in the reaction scheme reported in Figure 2A, the NPs are first dispersed in the organic phase of the water-in-oil microemulsion, obtained by mixing cyclohexane with suitable amount of Igepal CO-520 non-ionic surfactant and water ammonia solution. Upon addition of TEOS (Figure 2A step 1 and 2), surfactant molecules and TEOS remove the native organic ligands at the NP surface and such hydrophilized NPs can be entrapped within the water pool of the reverse micelle (Figure 2A step 3), the confined space where the formation of the siloxane network takes place (Figure 2A step 4).

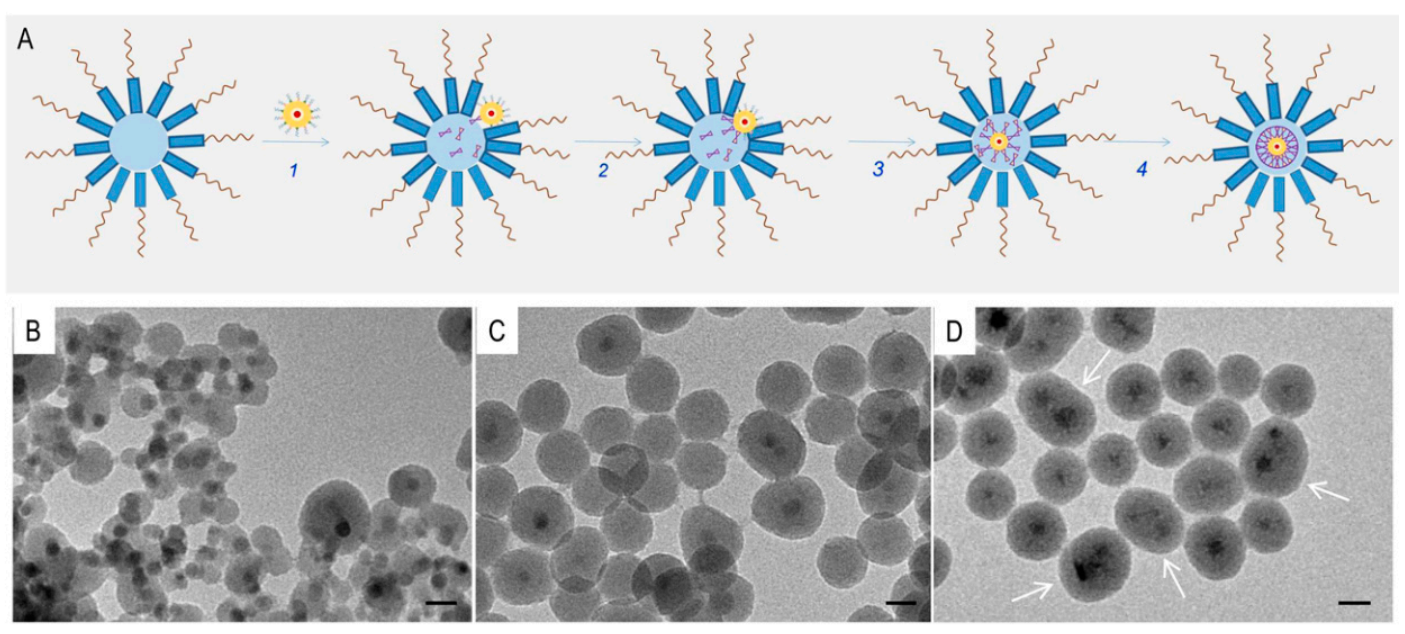

Figure 2. (A) Scheme of the water-in-oil microemulsion approach for silica shell growth: addition of the organic-capped giant quantum dots (QDs) to the water-in-oil microemulsion (step 1), displacement of the native ligands at the QD surface by surfactant molecules and hydrolyzed TEOS (step 2), inclusion of the surface modified QDs inside the water pool of the inverse micelle (step 3) and formation of the siloxane network around the QDs (step 4). TEM micrographs (Scale bar $50 \mathrm{~nm}$ ) of the silica nanostructures prepared using native "giant" quantum dots (GQDs) (B) and oleyl amine-treated GQDs at increasing volume of GQDs: $30 \mu \mathrm{L}(\mathbf{C})$ and $80 \mu \mathrm{L}(\mathbf{D})$. The reaction mixture consists of $350 \mu \mathrm{L}$ Igepal CO-520, $200 \mu \mathrm{L} \mathrm{NH}{ }_{4} \mathrm{OH}, 50 \mu \mathrm{L}$ TEOS and $30 \mu \mathrm{L}$ APTS. The reaction is let to proceed overnight at $28^{\circ} \mathrm{C}$ temperature. Arrow used in panel D to highlight the elongated multicore structures (32\% yield).

As demonstrated for CdSe [33,41,42,48], PbS [34] and metal NPs [32], the relatively mild conditions used for the functionalization and the transparency of the silica matrix in the regions of the electromagnetic spectrum where most of the NPs are photoactive, generally do not alter the NPs optical properties once the silica shell is grown. However, the geometry of the final nanostructures has been reported to critically affect the spectroscopic properties and/or the colloidal stability [32,33], 
making the careful control on the structure of the final product a challenging requirement. While single silica coated NPs have been found to preserve absorption and/or luminescence properties of the as prepared NPs, an increase in the NP core multiplicity in the final silica nanostructure has been reported to induce collective phenomena [32,49], such as absorption broadening, peak position shift or additional non radiative de-excitation pathways, resulting in partial luminescence quenching and reduced lifetimes $[48,50,51]$.

According to the mechanism that underpins the microemulsion synthetic method, the displacement of the native ligands at the QDs surface, which depends on the composition of the ligand passivating layer, ligand density, reactivity and kinetics of the ligand exchange reaction [52-54], regulates the NP encapsulation within the water droplet. Furthermore, at a fix Igepal-C0520 concentration, thus reverse micelle concentration, the relative NPs/TEOS ratio may affect the silica occupancy and core multiplicity within each silica NP.

Here, we performed a systematic control of the synthetic conditions, such as control of the ligand composition at the GQD surface by post synthesis ligand exchange reaction and of GQDs/TEOS molar ratio, to prevent the formation of multicore GQD silica shell structures, and, at the same time, to limit the formation of empty silica NPs that would reduce the yield of photoactive structures, tightly aggregated NPs and inhomogeneous silica shells [47,55].

Figure 2B-D displays the TEM micrographs of the silica nanostructures prepared from pristine (Figure 2B) and OLA-treated (Figure 2C,D) GQDs, at increasing GQD concentration and fixed surfactant $(350 \mu \mathrm{L})$ and TEOS volume $(50 \mu \mathrm{L})$. OLA has been selected for the post synthesis treatment due to its ability to passivate CdS of the GQD outer shell, but not too tightly coordinating the surface, thus enabling an easier subsequent ligand replacement.

Asymmetric (Janus type) and off-centered GQDs (Figure 2B) silica based nanostructures were synthesized starting from pristine GQDs $(30 \mu \mathrm{L})$, while isotropic core-shell NPs have been obtained when the GQDs have been pretreated with OLA (Figure 2C,D). A non-negligible percentage $(20 \%)$ of empty silica NPs was attained when using $30 \mu \mathrm{L}$ of the OLA-treated GQDs dispersion (Figure 2B) while, when a larger GQDs volume (up to $80 \mu \mathrm{L}$ ) was used, the amount of empty silica NPs effectively dropped and the procedure results essentially in core-shell type nanostructures (Figure 2C). In this latter case, elongated nanostructures (yield 32\%) hosting multiple but well separated GQDs are also detected, which turn into interconnected worm like structures, at further increasing of the GQDs concentration (data not shown).

To understand the mechanism that directs core-shell geometry, the role played by the GQD surface ligand stoichiometry and composition has to be considered. The outmost CdS shell, obtained by sulfur injection and dropwise addition of $\mathrm{Cd}(\mathrm{OA})_{2}$ in OLA and ODE, as reported in the Experimental section, can be described as $(\mathrm{CdS})_{\mathrm{n}}\left(\mathrm{Cd}(\mathrm{OA})_{2}\right)_{\mathrm{m}}(\mathrm{OLA})_{\mathrm{p}}$, where $n$ relates to the size of the shell and $m$ and $p$ describe the stoichiometry of the ligand shell, also based on the preservation of the GQD electroneutrality [54]. According to ligand classification described by Anderson et al. [56], OLA is an L-type ligand, which is usually not tightly bound to the surface, and $\mathrm{Cd}(\mathrm{OA})_{2}$ is a $\left(\mathrm{CdX}_{2}\right)$ Z-type ligand. Therefore, a mixed L- and Z-type surface chemistry is expected at the GQD surface. During the purification steps, ethanol added as non-solvent can easily remove OLA L-type ligands, while there is a limit to the amount of oleate X-type ligand that can be removed from the surface due to the charge arising from the excess of residual $\mathrm{Cd}^{2+}$ ions. Similarly, during the silica shell growth, TEOS and IGEPAL may preferentially exchange loosely bound OLA capping molecules rather than oleate ligand. Therefore, the mixed ligands composition at the surface of pristine GQDs results in a not homogenous silica shell. Conversely, the post synthesis OLA treatment promote the partial displacement of the $\mathrm{Cd}(\mathrm{OA})_{2} \mathrm{Z}$-type ligands by the excess of OLA added to the GQD sample, finally resulting in a $(\mathrm{CdS})_{\mathrm{n}}\left(\mathrm{Cd}(\mathrm{OA})_{2}\right)_{\mathrm{m}-\mathrm{x}}(\mathrm{OLA})_{\mathrm{p}+\mathrm{x}}$ surface stoichiometry that still preserves the GQD electroneutrality. As a result, a much more uniform OLA ligand layer coordinates the GQDs surface, thus driving the formation of a homogenous silica coating. In addition, the OLA left free in the microemulsion can be assumed to behave as a co-surfactant, lowering the interfacial surface 
tension and hence the reverse micellar film rigidity, thus energetically favoring the entrapping of the GQDs inside the water pool. The micelle occupancy also depends on the GQDs content. While empty silica NPs, together with core-shell structures, were formed using $30 \mu \mathrm{L}$ OLA-treated GQDs (Figure 2C), the increase in OLA-treated GQDs sample volume up to $80 \mu \mathrm{L}$ (Figure 2D) improved the yield of core-shell structures. Meanwhile, in this latter case, the excess of OLA, free in microemulsion, can promote micelles coalescence due to interfacial tension reduction, that template the formation of elongated silica structures hosting multiple well-separated cores (Figure 2D). The role of free OLA becomes more prominent further increasing the OLA-treated GQDs concentration, with the formation of worm like structures (data not shown).

Figure 3 displays TEM micrographs (Figure $3 \mathrm{~A}, \mathrm{~B}$ ) and the corresponding statistical analysis (Figure 3A1,B1) of single core nanostructures prepared using $80 \mu \mathrm{L}$ of OLA-treated GQDs and two different amounts of TEOS, $20 \mu \mathrm{L}$ (Figure 3A, sample GQDs@SiO $\mathrm{S}_{2} \mathrm{NP1}$ ) and $50 \mu \mathrm{L}$ (Figure 3B, sample GQDs@SiO $\left.{ }_{2} \mathrm{NP2}\right)$ respectively. GQDs@SiO ${ }_{2} \mathrm{NP1}$ has an average size of $33 \mathrm{~nm}(\sigma \%=8 \%)$ and a shell with an average thickness of $12 \mathrm{~nm}(\sigma \%=15 \%)$, while GQDs@SiO $\mathrm{NP}_{2}$ results in larger NPs (average size $38 \mathrm{~nm}, \sigma \%=11 \%$ ) characterized by a thicker silica shell $(15 \mathrm{~nm}, \sigma \%=13 \%)$ as expected from increasing the TEOS volume $(50 \mu \mathrm{L})$.
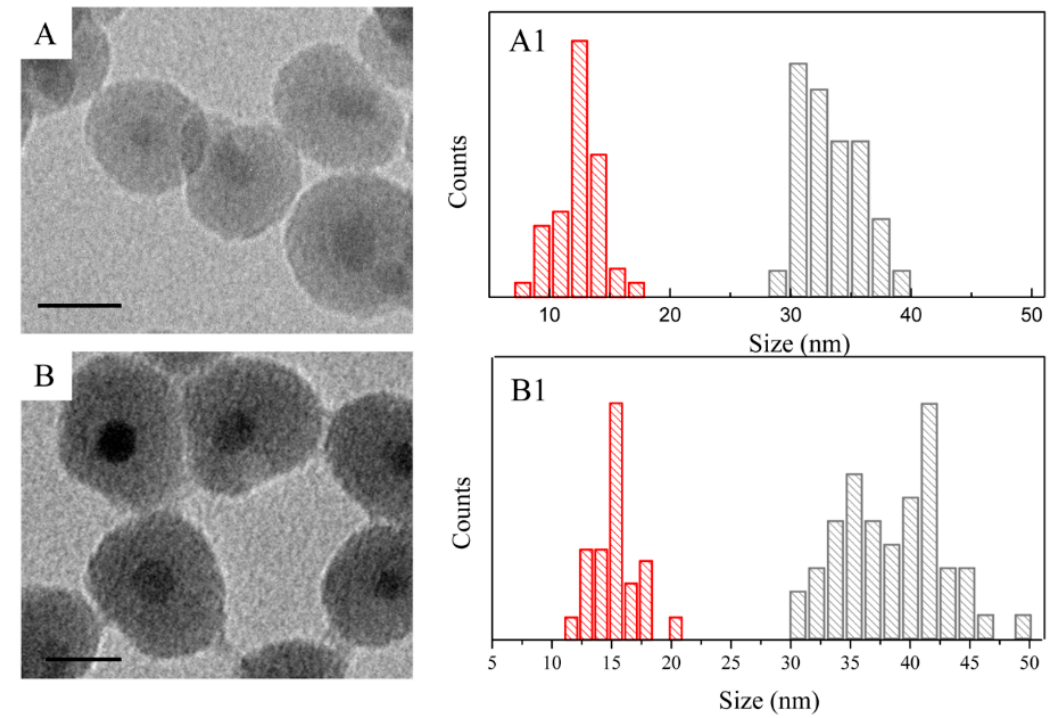

Figure 3. TEM images (A,B) and statistical analysis (A1,B1, performed on 200 nanoparticles (NPs) for each sample) of silica coated GQDs NPs (grey bar graph A1,B1) and silica shell (red bar graph A1,B1), prepared in a microemulsion at different TEOS volume: $20 \mu \mathrm{L}(\mathbf{A}, \mathbf{A 1})$ and $50 \mu \mathrm{L}(\mathbf{B}, \mathbf{B 1})$ of TEOS. Additionally, there were $80 \mu \mathrm{L}$ of OA-treated GQDs, $6 \mathrm{~mL}$ of cyclohexane, $350 \mu \mathrm{L}$ of Igepal CO-520 and $200 \mu \mathrm{L}$ of $\mathrm{NH}_{4} \mathrm{OH}$. The reaction has been let to proceed overnight at $28^{\circ} \mathrm{C}$. Scale bar $50 \mathrm{~nm}$.

In both these synthetic conditions, no appreciable changes in the core multiplicity can be observed, with the final NPs presenting the higher percentage (67\%) of monocore NP.

The highly monodispersed GQDs@SiO 2 NP1 sample was chosen as a model system to be functionalized with reactive anchoring amino groups by condensation reaction with APS and for further investigation of optical temperature-dependent measurements.

Functionalization of the silica NPs with APS leading to surface grafting of amino groups has been extensively investigated [48]. In the late stage of the silica shell growth suitable amount of APS was added and allowed to react overnight. After several purification steps and dispersion in water, concentration of the grafted amino groups was measured by the ninhydrin assay (see Materials and Methods), resulting in nearly $0.75 \mu \mathrm{mol} / \mathrm{mg}$. The chemical modification induced on the silica surface by grafted amino groups was also detected by means of $\xi$-potential measurements. GQDs@SiO $\mathrm{NPs}_{2}$ show a $\xi$-potential value of $-21( \pm 1) \mathrm{eV}$, due to the presence of negatively charged silanol groups, 
while a positive $\xi$-potential value $+20.0( \pm 0.4) \mathrm{eV}$ was measured for the amino-grafted GQDs@SiO ${ }_{2}$ $\mathrm{NP1}$, which was accounted by the successful grafting of positively charge amino moieties (pH6). The $\xi$-potential values of both the as prepared and amino-functionalized GQDs@SiO ${ }_{2} \mathrm{NP} 1$ indicate the good colloidal stability of both samples.

UV-Vis absorption and temperature dependent emission spectra of $\mathrm{GQDs}_{\mathrm{SSiO}} \mathrm{NP}_{2}$ are reported in Figure 4. The absorption spectrum (Figure 4A) clearly shows the characteristic absorption feature of the GQDs, although the exciton transition ascribed to the PbS core can be hardly observed, probably due to the high attenuation caused by the high extinction coefficient of the CdS and by scattering phenomena due to the silica shell. The temperature-dependent fluorescence of GQDs@SiO ${ }_{2} \mathrm{NP1}$ was explored by monitoring the emission (wavelength range of 450-750 nm) in the temperature range of 281-313 K, typical for biological applications [9], (Figure 4B). First, the emission spectra shows that, irrespectively from the temperature, the emission line profile is quite similar to that recorded for the GQDs [9]. Two emission bands, in the NIR region, ascribed to the PbS core and centered at $720 \mathrm{~nm}$, and in the visible range, related to the CdS shell, and centered at $480 \mathrm{~nm}$, respectively, are clearly detected. Both the emission bands are red shifted with respect to their position in the pristine GQDs (716 and 465, respectively), however a larger shift was observed for the CdS related signal.
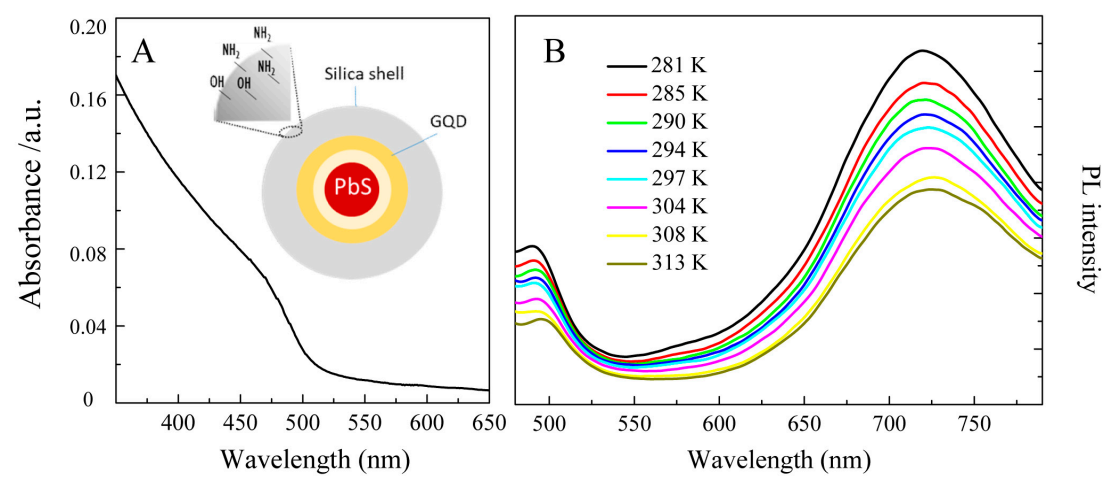

Figure 4. UV-Vis absorption (A) and fluorescence spectra in a $281-333 \mathrm{~K}$ temperature range $(\mathbf{B}, \lambda \mathrm{ex}=400 \mathrm{~nm})$ of silica coated GQDs NPs. A sketch of the nanostructure is shown in panel (A).

Such features can be described considering the structure of the GQDs, where the PbS core is expected to be nearly isolated from the environment due to the presence of the thick CdS shell. Conversely, the CdS shell is the outmost interface undergoing the whole procedure of silica shell formation (including the critical step of ligand exchange reaction). Therefore, the possible structural modification induced by the surface manipulation step-surface chemistry modification and change of the dielectric properties of the surrounding medium - may affect its optical properties. Beside the red shift of the CdS emission peak, the quenching of the CdS emission to a higher extent than $\mathrm{PbS}$ is also observed. The intensity ratio of the PbS emission band over the CdS emission band is 1.7 for GQDs at $294 \mathrm{~K}$, while it results 2.2 for the $\mathrm{GQDs}_{\mathrm{S}} \mathrm{SiO}_{2} \mathrm{NP}$ at the same temperature, thus confirming an effect of the silica shell growth on the optical properties of the CdS shell.

The temperature dependent fluorescence spectra reported in Figure $4 \mathrm{~B}$ were recorded in the temperature range purposely selected for biological applications. The results show that, as long as the temperature increased, a decrease of the luminescence intensity is observed, as also reported for the bare GQDs core [9].

Fitting each PL curve with a double Gaussian function corresponding to the CdS and PbS emission, respectively, and the integration of the photoluminescence contribution of both signals, allowed us to measure the integral area of the emission for each material ( $\mathrm{I}_{\mathrm{PbS}}$ and $\left.\mathrm{I}_{\mathrm{CdS}}\right)$ and, thus, to evaluate the ratiometric response in the temperature range investigated. Both the $\mathrm{PbS}$ (Figure $5 \mathrm{~A}$ red line and circle symbol) and the CdS (Figure 5A blue line and circle symbol) emission linearly decrease with temperature, with a more sensible emission quenching measured for the PbS signal. 

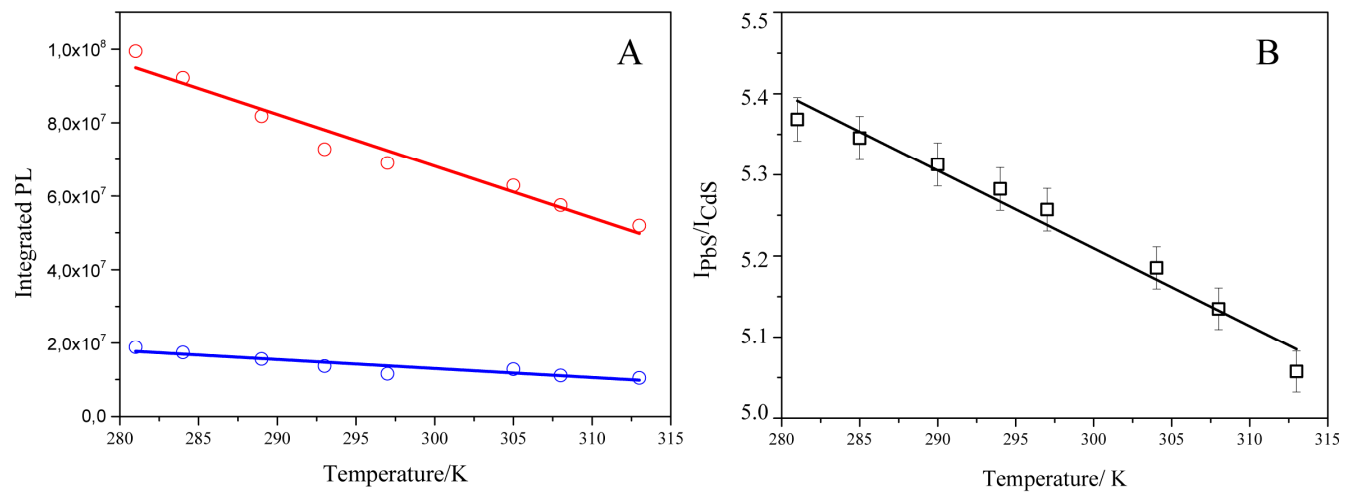

Figure 5. Scatter plot and line fit of (A) integrated fluorescence of CdS (blue symbol and line, $\mathrm{I}_{\mathrm{CdS}}$ ) and $\mathrm{PbS}$ (red symbol and line, $\mathrm{I}_{\mathrm{PbS}}$ ) versus temperature and (B) ratiometric response $\left(\mathrm{I}_{\mathrm{PbS}} / \mathrm{I}_{\mathrm{CdS}}\right)$ versus temperature.

This result can be explained by taking into account the material's thermal stability. The PbS core [9] are much more sensitive to temperature changes than the CdS, probably due to thermal activated traps/defects. The ratiometric response (Figure $5 \mathrm{~B}$ ) shows that $\mathrm{I}_{\mathrm{PbS}} / \mathrm{I}_{\mathrm{CdS}}$ monotonically decreased with temperature with a slope of $0.01 \mathrm{~K}^{-1}\left(\mathrm{R}^{2}=0.97\right)$, which coincides with that of the bare GQDs, in the same temperature range. The average relative sensitivity is nearly $0.2 \% \mathrm{~K}^{-1}$, higher than the value measured for non-ratiometric QDs [57] and within the range measured for rare earth doped NPs $\left(3.7-0.1 \% \mathrm{~K}^{-1}\right)[1,7,8,17,18]$. It has to be pointed out that the temperature response relies on mechanisms that are specific for each type of material, temperature range and environment; therefore, a direct comparison among diverse probes is not strictly relevant. In addition, by taking into account each system advantages and limitations, the availability of diverse temperature probes at the nanoscale offers the possibility to select the system most suitable for the specific case of study.

Overall, our results clearly indicate that the optical temperature-dependent response of the GQDs was not significantly affected by (i) the whole procedure developed to grow the silica coating, (ii) the presence of the obtained hydrophilic silica shell and (iii) dispersion in aqueous medium. Therefore, these features proved that the silica functionalization represents a reliable strategy to coat GQDs towards their effective use as biological/biomedical temperature probes.

\section{Conclusions}

Silica coated GQDs were prepared by means of a microemulsion method, purposely tailoring the synthetic protocol in order to achieve single core, homogenously coated core-shell structures, and limited empty silica NP formation. Control of synthetic parameters such as GQDs ligand composition and the GQDs/silica precursor molar ratio were demonstrated effective to obtain nanostructures able to retain the GQDs optical properties, and, specifically, the characteristic dual emission in the NIR and visible range, ascribed to the $\mathrm{PbS}$ core and the CdS giant shell, respectively. The temperature dependent fluorescence, previously demonstrated for bare GQDs, was proved also after silica shell growth, with a ratiometric response $\left(\mathrm{I}_{\mathrm{PbS}} / \mathrm{I}_{\mathrm{CdS}}\right)$ that monotonically decreased with the temperature, in temperature range suitable for biological systems $(281-313 \mathrm{~K})$ and a sensitivity of $0.01 \mathrm{~K}^{-1}$.

Author Contributions: E.F. and N.D. conceived and designed the experiments; E.F., H.Z. and S.D.Z. performed the experiments; E.F., H.Z., F.R., N.D., A.V. and M.S. analyzed the data; A.V., F.R., M.L.C. and M.S. contributed reagents/materials/analysis tools; E.F., H.Z., N.D., M.L.C. and M.S. wrote the paper. All authors have read and agreed to the published version of the manuscript.

Funding: F.R. is grateful to the Canada Research Chairs program for partial salary support and acknowledges NSERC for supporting part of the operating costs. A.V. acknowledges the Kempe Foundation and the Knut \& Alice Wallenberg Foundation for partial funding. E.F., N.D., M.L.C., M.S. are grateful to National Interuniversity 
Consortium of Materials Science and Technology (INSTM) and PON Project ARS01_00637 TARANTO for partial financial support.

Conflicts of Interest: The authors declare no conflict of interest.

\section{References}

1. Cao, S.; Zheng, J.; Zhao, J.; Yang, Z.; Shang, M.; Li, C.; Yang, W.; Fang, X. Robust and Stable Ratiometric Temperature Sensor Based on Zn-In-S Quantum Dots with Intrinsic Dual-Dopant Ion Emissions. Adv. Fun. Mater. 2016, 26, 7224-7233. [CrossRef]

2. Gao, H.; Kam, C.; Chou, T.Y.; Wu, M.-Y.; Zhao, X.; Chen, S. A simple yet effective AIE-based fluorescent nano-thermometer for temperature mapping in living cells using fluorescence lifetime imaging microscopy. Nanoscale Horiz. 2020, 5, 488-494. [CrossRef] [PubMed]

3. Ye, F.; Wu, C.; Jin, Y.; Chan, Y.-H.; Zhang, X.; Chiu, D.T. Ratiometric Temperature Sensing with Semiconducting Polymer Dots. J. Am. Chem. Soc. 2011, 133, 8146-8149. [CrossRef] [PubMed]

4. Vetrone, F.; Naccache, R.; Zamarrón, A.; Juarranz de la Fuente, A.; Sanz-Rodríguez, F.; Martinez Maestro, L.; Martín Rodriguez, E.; Jaque, D.; García Solé, J.; Capobianco, J.A. Temperature sensing using fluorescent nanothermometers. ACS Nano 2010, 4, 3254-3258. [CrossRef]

5. Wang, C.; Xu, R.; Tian, W.; Jiang, X.; Cui, Z.; Wang, M.; Sun, H.; Fang, K.; Gu, N. Determining intracellular temperature at single-cell level by a novel thermocouple method. Cell Res. 2011, 21, 1517-1519. [CrossRef]

6. Lee, J.; Kotov, N.A. Thermometer design at the nanoscale. Nano Today 2007, 2, 48-51. [CrossRef]

7. Mazza, M.M.A.; Cardano, F.; Cusido, J.; Baker, J.D.; Giordani, S.; Raymo, F.M. Ratiometric temperature sensing with fluorescent thermochromic switches. Chem. Commun. 2019, 55, 1112-1115. [CrossRef]

8. Du, S.; Yang, W. A broad-range temperature sensor dependent on the magnetic and optical properties of $\mathrm{SrF}_{2}: \mathrm{Yb}^{3+}, \mathrm{Ho}^{3+}$. Cryst. Eng. Commun. 2019, 21, 1452-1457.

9. Zhao, H.; Vomiero, A.; Rosei, F. Ultrasensitive, biocompatible, self-Calibrating, multiparametric Temperature sensors. Small 2015, 11, 5741-5746. [CrossRef]

10. Mazza, M.M.A.; Raymo, R. Structural designs for ratiometric temperature sensing with organic fluorophores. J. Mater. Chem. C 2019, 7, 5333-5342. [CrossRef]

11. Qiao, J.; Mu, X.; Qi, L. Construction of fluorescent polymeric nano-thermometers for intracellular temperature imaging: A review. Biosens. Bioelectron. 2016, 85, 403-413. [CrossRef] [PubMed]

12. Macairan, J.-R.; Jaunky, D.B.; Piekny, A.; Naccache, R. Intracellular ratiometric temperature sensing using fluorescent carbon dots. Nanoscale Adv. 2019, 1, 105-113. [CrossRef]

13. Maestro, L.M.; Jacinto, C.; Silva, U.R.; Vetrone, F.; Capobianco, J.A.; Jaque, D.; García Solé, J. CdTe quantum dots as nanothermometers: Towards highly sensitive thermal imaging. Small 2011, 7, 1774-1778. [CrossRef] [PubMed]

14. Zaini, M.S.; Kamarudin, M.A.; Chiyi, J.L.Y.; Ahmad, S.A.A.; Mohmad, A.R. Temperature and power dependence of photoluminescence in PbS quantum dots nanoparticles. Sains Malaysiana 2019, 48, 1281-1288. [CrossRef]

15. Matsuda, Y.; Torimoto, T.; Kameya, T.; Kameyama, T.; Kuwabata, S.; Yamaguchi, H.; Niimi, T. ZnS-AgInS 2 nanoparticles as a temperature sensor. Sens. Actuators B Chem. 2013, 176, 505-508. [CrossRef]

16. Liu, X.; Tang, X.; Hou, Y.; Wu, Q.; Zhang, G. Fluorescent nanothermometers based on mixed shell carbon nanodots. RSC Adv. 2015, 5, 81713-81722. [CrossRef]

17. Green, K.; Huang, K.; Pan, H.; Han, G.; Lim, S.F. Optical Temperature Sensing With Infrared Excited Upconversion Nanoparticles. Front. Chem. 2018, 6, 416. [CrossRef]

18. Cheng, Y.; Gao, Y.; Lin, H.; Huang, F.; Wang, Y. Strategy design for ratiometric luminescence thermometry: Circumventing the limitation of thermally coupled levels. J. Mater. Chem. C 2018, 6, 7462-7478. [CrossRef]

19. Uchiyama, S.; Matsumura, Y.; Prasanna de Silva, A.; Iwai, K. Fluorescent Molecular Thermometers Based on Polymers Showing Temperature-Induced Phase Transitions and Labeled with Polarity-Responsive Benzofurazans. Anal. Chem. 2003, 75, 5926-5935. [CrossRef]

20. Wang, C.; Otto, S.; Dorn, M.; Heinze, K.; Resch-Genger, U. Luminescent TOP nanosensors for simultaneously measuring temperature, oxygen, and $\mathrm{pH}$ at a Single Excitation Wavelength. Anal. Chem. 2019, 91, 2337-2344.

21. Resch-Genger, U.; Grabolle, M.; Cavaliere-Jaricot, S.; Nitschke, R.; Nann, T. Quantum dots versus organic dyes as fluorescent labels. Nat. Methods 2008, 5, 763-775. [CrossRef] [PubMed] 
22. Lutich, A.A.; Mauser, C.; Da Como, E.; Huang, J.; Vaneski, A.; Talapin, D.V.; Rogach, A.L.; Feldmann, J. Multiexcitonic Dual Emission in CdSe/CdS Tetrapods and Nanorods. Nano Lett. 2010, 10, 4646-4650. [CrossRef] [PubMed]

23. Choi, L.C.; Li, H.; Olson, A.C.K.; Jain, P.K.; Sivasankar, S.; Alivisatos, A.P. Spatially Indirect Emission in a Luminescent Nanocrystal Molecule. Nano Lett. 2011, 11, 2358-2362. [CrossRef] [PubMed]

24. Galland, C.; Brovelli, S.; Bae, W.K.; Padilha, L.A.; Meinardi, F.; Klimov, V.I. Dynamic Hole Blockade Yields Two-Color Quantum and Classical Light from Dot-in-Bulk Nanocrystals. Nano Lett. 2013, 13, 321-328. [CrossRef] [PubMed]

25. Cirloganu, C.M.; Padilha, L.A.; Lin, Q.; Makarov, N.S.; Velizhanin, K.A.; Luo, H.; Robel, I.; Pietryga, J.M.; Klimov, V.I. Enhanced carrier multiplication in engineered quasi-type-II quantum dots. Nat. Commun. 2014, 5, 4148. [CrossRef]

26. Zhao, H.; Sirigu, G.; Parlsini, A.; Camellini, A.; Nicotra, G.; Rosei, F.; Morandi, V.; Zavelani-Rossi, M.; Vomiero, A. Dual emission in asymmetric "giant" PbS/BdS/BdS core/shell/shell quantum dots. Nanoscale 2016, 8, 4217-4226. [CrossRef]

27. Navarro-Parso, F.; Zhao, H.; Wang, M.Z.; Rosei, F. Structure/property relations in "giant" semiconductor Nanocrystals: Opportunities in photonics and electronics. Acc. Chem. Res. 2018, 51, 609-618. [CrossRef]

28. Liu, J.; Zhang, H.; Selopal, G.S.; Sun, S.; Zhao, H.; Rosei, F. Visible and near-infrared, multiparametric, ultrasensitive nanothermometer based on dual-emission colloidal quantum dots. ACS Photonics 2019, 6, 2479-2486. [CrossRef]

29. Latronico, T.; Depalo, N.; Valente, G.; Fanizza, E.; Laquintana, V.; Denora, N.; Fasano, A.; Striccoli, M.; Colella, M.; Agostiano, A.; et al. Cytotoxicity study on luminescent nanocrystals containing phospholipid micelles in primary cultures of rat astrocytes. PLoS ONE 2016, 11, e0153451. [CrossRef]

30. Depalo, N.; De Leo, V.; Corricelli, M.; Gristina, R.; Valente, G.; Casamassima, E.; Comparelli, R.; Laquintana, V.; Denora, N.; Fanizza, E.; et al. Lipid-based systems loaded with PbS nanocrystals: Near infrared emitting trackable nanovectors. J. Mater. Chem. B 2017, 5, 1471-1481. [CrossRef]

31. Zhang, F.; Lees, E.; Amin, F.; Rivera Gil, P.; Yang, F.; Mulvaney, P.; Parak, W.J. Polymer-Coated Nanoparticles: A Universal Tool for Biolabelling Experiments. Small 2011, 7, 3113-3127. [CrossRef] [PubMed]

32. Fanizza, E.; Depalo, N.; Clary, L.; Agostiano, A.; Striccoli, M.; Curri, M.L. A combined size sorting strategy for monodisperse plasmonic nanostructured. Nanoscale 2013, 5, 3272-3282. [CrossRef] [PubMed]

33. Fanizza, E.; Urso, C.; Pinto, V.; Cardone, A.; Depalo, N.; Ragni, R.; Curri, M.L.; Agostiano, A.; Farinola, G.M.; Striccoli, M. Single White Light Emitting Hybrid Nanoarchitectures Based on Functionalized Quantum Dots. J. Mater. Chem. C 2014, 2, 5286-5291. [CrossRef]

34. Corricelli, M.; Depalo, N.; Di Carlo, E.; Fanizza, E.; Laquintana, V.; Denora, N.; Agostiano, A.; Striccoli, M.; Curri, M.L. Biotin-decorated silica coated PbS nanocrystals emitting in the second biological near infrared window for bioimaging. Nanoscale 2014, 6, 7924-7933. [CrossRef]

35. Jun, B.-H.; Hwang, D.W.; Jung, H.S.; Jang, J.; Kim, H.; Kang, H.; Kang, T.; Kyeong, S.; Lee, H.; Jeong, D.H.; et al. Ultrasensitive, Biocompatible, Quantum-Dot-Embedded Silica Nanoparticles for Bioimaging. Adv. Funct. Mater. 2012, 22, 1843-1849. [CrossRef]

36. Ruan, J.; Wang, K.; Song, H.; Xu, X.; Ji, J.; Cui, D. Biocompatibility of hydrophilic silica-coated CdTe quantum dots and magnetic nanoparticles. Nanoscale Res. Lett. 2011, 6, 299. [CrossRef]

37. Yang, P.; Murase, N.; Suzuki, M.; Hosokawa, C.; Kawasaki, K.; Kato, T.; Taguchi, T. Bright, non-blinking, and less-cytotoxic $\mathrm{SiO}_{2}$ beads with multiple CdSe/ZnS nanocrystals. Chem. Commun. 2010, 46, 4595-4597. [CrossRef]

38. Cendrowski, K.; Sikora, P.; Zielinska, B.; Horszczaruk, E.; Mijowska, E. Chemical and thermal stability of core-shelled magnetite nanoparticles and solid silica. Appl. Surf. Sci. 2017, 407, 391-397. [CrossRef]

39. Savchuk, O.A.; Carvajal, J.J.; Cascales, C.; Aguilò, M.; Diaz, F. Benefits of Silica Core-Shell Structures on the Temperature Sensing Properties of Er, $\mathrm{Yb}: \mathrm{GdVO}_{4} \mathrm{Up}$-Conversion Nanoparticles. ACS Appl. Mater. Interfaces 2016, 8, 7266-7273. [CrossRef]

40. Zhao, H.; Wu, N.; Chaker, M.; Ma, D. Towards controlled synthesis and better understanding of highly luminescent PbS/CdS core/shell quantum dots. J. Mater. Chem. 2011, 21, 8898-8904. [CrossRef]

41. Pati, M.L.; Fanizza, E.; Hager, S.; Groza, D.; Heffeter, P.; Laurenza, A.G.; Laquintana, V.; Curri, M.L.; Depalo, N.; Abate, C.; et al. Quantum Dot Based Luminescent Nanoprobes for Sigma-2 Receptor Imaging. Mol. Pharm. 2018, 15, 458-471. 
42. Fanizza, E.; Iacobazzi, R.M.; Laquintana, V.; Valente, G.; Caliandro, G.; Striccoli, M.; Agostiano, A.; Cutrignelli, A.; Lopedota, A.; Curri, M.L.; et al. Highly selective luminescent nanostructures for mitochondria imaging and targeting. Nanoscale 2016, 8, 3350-3361. [CrossRef] [PubMed]

43. Fanizza, E.; Depalo, N.; Fedorenko, S.; Iacobazzi, R.M.; Mukhametshina, A.; Zairov, R.; Salatino, A.; Vischio, F.; Panniello, A.; Laquintana, V.; et al. Green fluorescent terbium (III) complex doped silica nanoparticles for TSPO targeting. Int. J. Mol. Sci. 2019, 20, 3139. [CrossRef] [PubMed]

44. Kang, D.; Kumar, M.B.; Son, C.; Park, H.; Park, J. Simple synthesis Method and Characterizations of Aggregation-Free Cysteamine Capped PbS Quantum Dot. Appl. Sci. 2019, 9, 4661. [CrossRef]

45. Vischio, F.; Fanizza, E.; Debellis, V.; Sibbillano, T.; Ingrosso, C.; Giannini, C.; Laquintana, V.; Denora, N.; Agostiano, A.; Striccoli, M.; et al. Near-Infrared absorbing solid lipid nanoparticles encapsulating plasmonic copper sulfide nanocrystals. J. Phys. Chem. C 2019, 123, 23205-23213. [CrossRef]

46. Yang, S.-A.; Choi, S.; Jeon, S.M.; Yu, J. Silica nanoparticle stability in biological media revisited. Sci. Rep. 2018, 8, 185. [CrossRef]

47. Dávila-Ibáñez, A.B.; Mariño-Fernández, R.; Maceira-Campos, M.; García-Lorenzo, A.; Martínez-Zorzano, V.; Salgueiriño, V. Nonhomogeneous silica promotes the biologically induced delivery of metal ions from silica-coated magnetic nanoparticles. J. Phys. Chem. C 2014, 118, 28266-28273.

48. Fanizza, E.; Urso, C.; Iacobazzi, R.M.; Depalo, N.; Corricelli, M.; Panniello, A.; Agostiano, A.; Denora, N.; Laquintana, V.; Striccoli, M.; et al. Fabrication of photoactive heterostructures based on quantum dots decorated with Au nanoparticles. STAM 2016, 17, 98-108. [CrossRef]

49. Wang, H.; Schaefer, K.; Moeller, M. In situ Immobilization of gold nanoparticle dimers in silica nanoshell by microemulsion coalescence. J. Phys. Chem. Lett. C 2008, 112, 3175-3178. [CrossRef]

50. Cui, J.; Panfil, Y.E.; Koley, S.; Shamalia, D.; Waiskopf, N.; Remennik, S.; Popov, I.; Oded, M.; Banin, U. Colloidal quantum dot molecules manifesting quantum coupling at room temperature. Nat. Commun. 2019, 10, 5401. [CrossRef]

51. Dibenedetto, C.N.; Fanizza, E.; Brescia, R.; Kolodny, Y.; Remennik, S.; Panniello, A.; Depalo, N.; Yochelis, S.; Comparelli, M.; Agostiano, A.; et al. Coupling effects in QD dimers at sub-nanometer interparticle distance. Nano Res. 2020. [CrossRef]

52. Liu, M.; Wang, Y.-Y.; Liu, Y.; Jiang, F.-L. Thermodynamic implications of the ligand exchange with alkylamines on the surface of CdSe quantum dots: The importance of ligand-ligand interactions. J. Phys. Chem. C 2020, 124, 4613-4625. [CrossRef]

53. Shakeri, B.; Meulenberg, R.W. A closer look into the traditional purification process of CdSe semiconductor quantum dots. Langmuir 2015, 31, 13433-13440. [CrossRef] [PubMed]

54. Boles, M.A.; Ling, D.; Hyeon, T.; Talapin, D.V. The surface science of nanocrystals. Nat. Mater. 2016, 15, 141-153. [CrossRef] [PubMed]

55. Hlaváček, A.; Sedlmeier, A.; Skládal, P.; Gorris, H.H. Electrophoretic Characterization and purification of silica-coated photon-upconverting nanoparticles and their bioconjugates. ACS Appl. Mater. Interfaces 2014, 6, 6930-6935. [CrossRef] [PubMed]

56. Anderson, N.C.; Hendricks, M.P.; Choi, J.J.; Owen, J.S. Ligand exchange and the stoichiometry of metal chalcogenide nanocrystals: Spectroscopic observation of facile metal-carboxylate displacement and binding. J. Am. Chem. Soc. 2013, 135, 18536-18548. [CrossRef]

57. Choudhury, D.; Jaque, D.; Rodenas, W.T.; Ramsey, T.; Paterson, L.; Kar, A.K. Quantum dot enabled thermal imaging of optofluidic devices. Lab Chip 2012, 12, 2414-2420. [CrossRef]

(C) 2020 by the authors. Licensee MDPI, Basel, Switzerland. This article is an open access article distributed under the terms and conditions of the Creative Commons Attribution (CC BY) license (http://creativecommons.org/licenses/by/4.0/). 